\title{
Análisis crítico de un artículo: «Impacto de la oximetría fetal en la tasa de cesáreas»
}

\author{
Jaime Cerda L ${ }^{1,2, a}$, Claudio Vera P-G ${ }^{1,3}$. \\ Critically appraised article: \\ Fetal pulse oximetry and cesarean \\ delivery. \\ N Engl J Med 2006; 355: 2195-202. \\ Bloom S, Spong C, Thom E, \\ Varner $M$, Rouse $D$, Weininger, et al.
}

\begin{abstract}
Background: Knowledge of fetal oxygen saturation, as an adjunct to electronic fetal monitoring, may be associated with a significant change in the rate of cesarean deliveries or the infant's condition at birth. Methods: We randomly assigned 5341 nulliparous women who were at term and in early labor to either «open» or «masked» fetal pulse oximetry. In the open group, fetal oxygen saturation values were displayed to the clinician. In the masked group, the fetal oxygen sensor was inserted and the values were recorded by computer, but the data were hidden. Labor complicated by a nonreassuring fetal heart rate before randomization was documented for subsequent analysis. Results: There was no significant difference in the overall rates of cesarean delivery between the open and masked groups $(26.3 \%$ and $27.5 \%$, respectively; $\mathrm{p}=0.31)$. The rates of cesarean delivery associated with the separate indications of a nonreassuring fetal heart rate $(7.1 \%$ and $7.9 \%$, respectively; $\mathrm{p}=0.30)$ and dystocia ( $18.6 \%$ and $19.2 \%$, respectively; $\mathrm{p}=0.59)$ were similar between the two groups. Similar findings were observed in the subgroup of 2168 women in whom a nonreassuring fetal heart rate was detected before randomization. The condition of the infants at birth did not differ significantly between the two groups. Conclusions: Knowledge of the fetal oxygen saturation is not associated with a reduction in the rate of cesarean delivery or with improvement in the condition of the newborn.
\end{abstract}

\section{Conclusión de los ReVisores:}

El manejo del trabajo de parto basado en la monitorización continua de oximetría de pulso fetal asociado a cardiotocografía no reduce la tasa de cesáreas ni mejora la condición del recién nacido comparado con el manejo basado sólo en cardiotocografía.

\footnotetext{
${ }^{1}$ Unidad de Medicina Basada en Evidencia. Facultad de Medicina, Pontificia Universidad Católica de Chile.

${ }^{2}$ Departamento de Salud Pública. Facultad de Medicina, Pontificia Universidad Católica de Chile.

${ }^{3}$ Departamento de Obstetricia y Ginecología. Facultad de Medicina, Pontificia Universidad Católica de Chile. Santiago de Chile.

${ }^{\text {aResidente. }}$
} 


\section{PREgUNTA CLÍNICA:}

En el manejo del trabajo de parto ¿es posible reducir la tasa de cesáreas y las complicaciones del recién nacido con el uso de monitorización continua de la oximetría fetal como adyuvante a la cardiotocografía comparada con el manejo basado sólo en cardiotocografía?

\section{ConTEXTO:}

La monitorización continua de la frecuencia cardíaca fetal y de las contracciones uterinas (cardiotocografía) es el examen más frecuentemente utilizado para detectar compromiso fetal durante el trabajo de parto. Su implementación no ha estado exenta de controversia ${ }^{1}$, pues se ha propuesto que la baja reproducibilidad y alta tasa de falsos positivos de este examen contribuyen al incremento de la tasa de cesáreas ${ }^{2}$. En el año 2000, la FDA (Food and Drug Administration) aprobó de manera condicional el uso de la monitorización de oximetría fetal como adyuvante a la monitorización cardiotocográfica. La monitorización de oximetría fetal fue diseñada con el objetivo de mejorar el conocimiento de la condición fetal a través de la medición continua de la saturación de oxígeno en presencia de un patrón de frecuencia cardíaca fetal anormal y así lograr disminuir el número de falsos positivos de la cardiotocografía $^{3}$. La aprobación condicional de la monitorización de oximetría fetal se basó en un estudio randomizado controlado que mostró una reducción significativa de la tasa de cesáreas indicada por una frecuencia cardíaca fetal anormal $(10,2 \% \text { a 4,5\%) })^{4}$. Sin embargo, la tasa de cesáreas por distocia en el grupo en que se utilizó oximetría fetal como adyuvante duplicó a la del grupo control, sin lograrse una disminución de la tasa de cesárea total. Por consiguiente, el presente estudio se realizó con el objetivo de aportar nueva evidencia que permita recomendar o rechazar la implementación de esta estrategia adyuvante de monitorización fetal.

\section{Características del ESTUdio:}

Tipo de estudio: randomizado, multicéntrico. Pacientes: 5.341 mujeres nulíparas, cursando embarazo único con feto vivo en presentación cefálica y dilatación cervical entre 2-6 cm. Se excluyeron pacientes con cesárea programada, fiebre materna, infección conocida por VIH o hepatitis, enfermedad cardíaca o renal y diabetes mellitus. Intervención: 2.629 embarazadas sometidas a monitorización de oximetría fetal (abierta al obstetra tratante) más monitorización cardiotocográfica. Comparación: 2.712 embarazadas sometidas a monitorización de oximetría fetal (oculta al obstetra tratante) más monitorización cardiotocográfica. Outcome Primario: tasa de cesárea. OutcomeSecundario: complicaciones neonatales (outcome compuesto por uno o más de los siguientes: puntaje APGAR a los 5 minutos inferior a 4, pH de sangre de arteria umbilical bajo 7,0 , convulsiones, intubación en la sala de parto, mortinato, muerte neonatal e ingreso a unidad de cuidados intensivos neonatales por más de 48 horas). Seguimiento: No especificado.

VALIDEZ:

\begin{tabular}{|ll|}
\hline Randomizado: & Sí \\
Ocultamiento de la secuencia de randomización: & No especificado \\
Seguimiento ${ }^{\text {: }}$ & $100 \%$ (outcome primario) \\
Análisis por intención de tratar: & Sí \\
Fueron ciegos a la intervención : & Sí \\
- Pacientes: & No ${ }^{b}$ \\
- Tratantes: & Sí \\
- Recolectores de datos: & Sí \\
- Adjudicadores de eventos: & No claro \\
- Analistas de datos: & Sí \\
\hline
\end{tabular}

${ }^{\mathrm{a}}$ Outcome secundario con seguimiento incompleto: datos del $\mathrm{pH}$ de arteria umbilical faltaron para 361 neonatos en el grupo abierto y 385 en el grupo control. bLos médicos tratantes conocían la asignación del binomio madre-hijo según la condición de monitorización de oximetría fetal (abierta = grupo experimental, oculta = grupo control). 
Resultados:

\begin{tabular}{|lccc|}
\hline Eventos & RR (95\% IC) & RRA (95\% IC) & NNT (95\% IC) \\
Cesáreas & $0,96(0,87$ a 1,04$)$ & $1,2(-1,16$ a 3,60) & 82 (28 a -86) \\
Complicaciones neonatales & $0,95(0,71$ a 1,27$)$ & $0,2(-0,79$ a 1,11$)$ & $624(90$ a -126$)$ \\
\hline
\end{tabular}

RR: riesgo relativo; RRA: reducción de riesgo absoluto; NNT: número necesario de tratar.

\section{COMENTARIOS Y APLICACIÓN PRÁCTICA:}

- El presente estudio cumple en una alta proporción los criterios de validez interna, permitiendo calificar su metodología como satisfactoria.

- Destaca positivamente el uso de ciegos, sobre todo en los recolectores de datos y adjudicadores de eventos, en situaciones en que los tratantes no pueden ser ciegos.

- Los resultados de la investigación no demuestran una reducción significativa de la tasa de cesárea ni una mejoría en la condición del recién nacido asociado al conocimiento del resultado de la oximetría de pulso además de la cardiotocografía para la evaluación fetal en el trabajo de parto. Estos resultados son concordantes con la revisión sistemática de los estudios randomizados previos disponible en la base de datos Cochranes.

- Es importante consignar que el estudio fue detenido precozmente (i.e. antes de completar el tamaño muestral planificado), hecho que pudiese haber ocultado el efecto real de la intervención.

- Una de las formas de estimar el riesgo neonatal por parte del equipo investigador fue utilizar un outcome compuesto, el cual homologa outcomes biofísicos y clínicos (e.g. $\mathrm{pH}$ de sangre en arteria umbilical y muerte neonatal). A pesar de no haber una diferencia significativa en la ocurrencia de este outcome entre los grupos experimental y control, es importante consignar que la naturaleza y relevancia de las variables que componen este outcome no son comparables.

- En relación a su aplicabilidad, la suma de métodos diagnósticos propuestos aún no logra la capacidad diagnóstica suficiente para reducir intervenciones innecesarias y mejorar el resultado neonatal. Mejores métodos deben ser desarrollados y evaluados rigurosamente antes de su incorporación a la práctica clínica.

\section{REFERENCIAS}

1. Intrapartum fetal heart rate monitoring. ACOG practice bulletin no. 70. Washington, DC: American College of Obstetricians and Gynecologists, December 2005.

2. Hamilton Be, Martin Ja, Ventura SJ, Sutton PD, Menacker F. Births: preliminary data for 2004. National vital statistics reports. Vol. 54. №. 8. Hyattsville, MD: National Center for Health Statistics, 2005.

3. Yam J, Chua S, Arulkumaran S. Intrapartum fetal pulse oximetry. Part I: principles and technical issues. Obstet Gynecol Surv 2000; 55: 163-72.

4. Garite TJ, Dildy Ga, McNamara H, Nagaotte MP Boehm FH, Dellinger EH et al. A multicenter controlled trial of fetal pulse oximetry in the management of nonreassuring fetal heart rate patterns. Am J Obstet Gynecol 2000; 185: 771-2.

5. East CE, Chan FY, Colditz PB, Begg LM. Fetal pulse oximetry for fetal assessment in labour. Cochrane Database of Systematic Reviews 2007, Issue 2. Art. No.: CD004075. 